\title{
Shock-Induced Flow Separation in an Overexpanded Supersonic Planar Nozzle
}

\author{
B. Zebiri,,- A. Piquet,, \pm and A. Hadjadj \\ University of Rouen, 76000 Rouen, France \\ and \\ S. B. Verma $\$$ \\ Council of Scientific and Industrial Research-National Aerospace Laboratories, \\ Bangalore 560 017, India \\ https://doi.org/10.2514/1.J058705
}

\begin{abstract}
Shock-induced flow separation in an overexpanded supersonic planar nozzle is investigated numerically by means of three-dimensional wall-modeled large-eddy simulations (LES). The objective of this study is to identify the origin of the low-frequency shock oscillations (LFO) and the associated side-loads generation in planar nozzles. The computational results are compared with the experimental data for validation. The promise of the near-wall LES modeling approach, adopted in this study, is supported by its satisfactory performance in correctly predicting the shock-induced flow separation and offering a major advantage of being 30-40 times faster than the wall-resolved LES counterpart, allowing thereby the capture of very-low-frequency shock oscillations with much better statistics convergence. The simulations bring clear evidence of the existence of broadband and energetically significant LFO in the vicinity of the separated shock, whose forward and backward movements are mainly driven by changes in the downstream flow conditions. The complex interactions between the backflow, the separation bubbles, and the large-scale turbulent structures developing in the shear-layer region strongly influence the shock unsteadiness, which in turn drives the LFO. A scenario of the LFO, confirming conclusions from earlier studies, is described in this work.
\end{abstract}

$\begin{array}{lll} & & \text { Nomenclature } \\ A & = & \text { nozzle effective area } \\ c & = & \text { speed of sound } \\ E & = & \text { total energy } \\ F & = & \text { force } \\ H_{t} & = & \text { nozzle-throat height } \\ L & = & \text { characteristic length } \\ M & = & \text { Mach number } \\ p & = & \text { static pressure } \\ Q_{j} & = & \text { subgrid heat flux } \\ \dot{q}_{j} & = & \text { heat-diffusion flux } \\ R_{\alpha \alpha} & = & \text { two-point correlation function } \\ S t & = & \text { Strouhal number } \\ T & = & \text { temperature } \\ t & = & \text { time } \\ u_{i} & = & \text { velocity components } \\ x, y, z & =\text { Cartesian coordinates } \\ \delta & = & \text { boundary-layer thickness } \\ \delta_{i j} & = & \text { Kronecker symbol } \\ \lambda & = & \text { thermal conductivity } \\ \mu & = & \text { dynamic viscosity } \\ \nu & = & \text { kinematic viscosity } \\ \xi, \eta, \zeta & =\text { generalized curvilinear coordinates }\end{array}$

Received 5 June 2019; revision received 3 December 2019; accepted for publication 10 December 2019; published online 13 February 2020. Copyright $\odot 2019$ by the American Institute of Aeronautics and Astronautics, Inc. All rights reserved. All requests for copying and permission to reprint should be submitted to CCC at www.copyright.com; employ the eISSN 1533-385X to initiate your request. See also AIAA Rights and Permissions www.aiaa.org/ randp.

*Ph.D. Student, National Institute of Applied Sciences, Centre National de la Recherche Scientifique, Unité Mixte de Recherche 6614, Avenue de l’Université; also CORIA INSA de Rouen; boubakr.zebiri@insa-rouen.fr.

${ }^{\dagger}$ Research Engineer, CORIA INSA de Rouen; arthur.piquet@ intradef.gouv.fr.

*Professor, National Institute of Applied Sciences, Centre National de la Recherche Scientifique, Unité Mixte de Recherche 6614, Avenue de l'Université; also CORIA INSA de Rouen; abdellah.hadjadj@insa-rouen.fr.

${ }^{\S}$ Deputy Head, Experimental Aerodynamics Division, and Group Head, Flow Structure Management and Aircraft and Spacecraft Aerodynamics; sbverma@nal.res.in. Associate Fellow AIAA.

$$
\begin{array}{ll}
\rho & =\text { density } \\
\sigma_{i j} & =\text { viscous-stress tensor } \\
\tau_{i j} & =\text { subgrid shear-stress tensor }
\end{array}
$$

\section{Subscripts}

$\begin{array}{lll}a & = & \text { ambient conditions } \\ e & = & \text { exit-nozzle variable } \\ i & = & \text { inlet-nozzle variable } \\ r & = & \text { resonant tone period } \\ \mathrm{rms} & = & \text { root mean square } \\ s & = & \text { separation-point variable } \\ \mathrm{sgs} & = & \text { subgrid scale } \\ \text { th } & = & \text { throat-based variable } \\ w & = & \text { wall variable } \\ \tau & = & \text { friction variable } \\ 0 & = & \text { total/stagnation variable } \\ \infty & = & \text { freestream condition }\end{array}$

Superscripts

$\begin{array}{lll}+ & = & \text { wall-units variable } \\ , & = & \text { fluctuating quantity } \\ \star & = & \text { normalized quantity }\end{array}$

\section{Introduction}

Q HOCK-INDUCED boundary-layer separation in transonic and $\checkmark$ supersonic flows is of great importance in many practical applications, such as rocket propulsion systems, transonic airfoils, and refrigeration ejectors. These unsteady phenomena are associated with undesirable effects such as shock oscillations, wall-pressure fluctuations, boundary-layer separation, and vortex shedding, which are major causes of vibration, noise, and side-loads generation. Comprehensive reviews of the topic can be found in [1].

Flow separation in supersonic planar nozzles has been the subject of several experimental and numerical investigations, with the aim to understand the different shock patterns occurring in the overexpanded nozzle regime [2-13]. According to these studies, three typical flow separation configurations can be identified: 1) incipient boundarylayer separation with normal shock, 2) symmetric boundary-layer 
separation with two-symmetric $\lambda$ shocks, and 3) asymmetric boundary-layer separation with two-asymmetric $\lambda$ shocks (see Fig. 1). As for the separation location, it was found to be sensitive to the flow disturbances, which may originate from the roughness of the wall [12] or from the improper initialization or startup of the flow [13]. To address the problem of nozzle flow separation, Papamoschou and Johnson [2] investigated a planar nozzle experimentally with a flexible geometry and different nozzle pressure ratios (NPR). They found that the flow could be asymmetric without flipping during the test run, but the jet flow could change the direction from one nozzle side to another during a restart of a run. According to their study, the shock selects its orientation at the nozzle startup and retains it throughout the run. Additionally, Reynolds-averaged Navier-Stokes (RANS) simulations were performed by Xiao et al. [3], in which they roughly confirmed the experimental findings of $[\overline{2}]$. For a fixed nozzle area ratio and over a wide range of NPR (1.269-2.4), they found that the flow can be asymmetric for NPR 1.5-2.4, which is consistent with the experiments of [2]. Xiao et al. [3] demonstrated that the asymmetric behavior of the flow separation could be forced on either the top or the bottom wall by means of perturbations placed near one of the nozzle walls. They also found that the separated-flow region is largely enhanced within the core of the nozzle flow, which exhibits a higher level of unsteadiness. Detailed investigations of flow separations were made by Hunter [4], who studied experimentally and numerically an overexpanded planar nozzle and found that the flow is mainly dominated by the shock-boundary-layer interaction dynamics. He proposed a scenario in which the separation could be a natural response of the flow in its tendency to reach a thermodynamically stable state. Based on his study, the author suggested to classify the separation into two categories according to the NPR: 1) a nonstationary three-dimensional separation with a partial re-attachment and 2) a stationary two-dimensional separation with an abrupt transition existing between the two. The origin of the flow asymmetry was also investigated experimentally by Verma and Manisankar [5] using three different Mach 2 planar nozzles with a fixed area ratio and different divergent-wall angles. In their study, the authors found that the boundary-layer transition is one of the main reasons for the flow asymmetry. They also showed that the transition from free-shock separation (FSS) to restricted-shock separation (RSS) could initiate the flow asymmetry, but later the transition from RSS to FSS seems to make the flow symmetric again. Another origin of asymmetry, reported by []], was the proximity of the separated shear layer to the nozzle wall.

In terms of unsteadiness, the separated flow often induces shock oscillations, associated with low-frequency motions. Two main origins of low-frequency shock oscillation (LFO) mechanisms can be broadly reported: The first concerns the upstream influence of the incoming turbulent boundary layer [14], whereas the second one (which is the most consensual today) supports the idea of the influence of the downstream conditions via the interaction between the separated boundary layer, the recirculation bubble, and the shocktrain system $[6-11,15,16]$. Obviously, The ultimate question is the following: According to which physical mechanisms can these low-frequency phenomena occur? Many scenarios are proposed in the literature. For instance, Zaman et al. [15], in their theoretical and experimental studies of transonic diffusers, concluded that the mechanism creating LFO is stimulated by acoustic resonance, where the low-frequency mode corresponds to the case when the onequarter wavelength is fitted within the approximate distance from the foot of the shock to the nozzle exit. The same conclusions were made by Johnson and Papamoschou [7] when investigating experimentally the flow in a supersonic planar nozzle. Olson and Lele []] investigated numerically the same nozzle and confirmed the previous findings. An interesting study of the effects of forced downstream conditions in a diffuser was conducted by Bruce and Babinsky [17]. The authors have found that the normal shock undergoes a coherent and reproducible periodic motion with regard to the sinusoidal variations of the downstream pressure. They also proposed a model for the trajectory of the shock that can be easily calculated by integrating the already predictable shock velocity. Thus, they define the shock movement as a mechanism by which the shock system can adapt itself to satisfy the NPR change. As a result, the frequency of the disturbance has no effect on the shock velocity. The effect of geometry was also studied through a nonviscous one-dimensional model. The authors were able to define a critical frequency, beyond which the amplitude of the shock is largely dependent on the angle of the divergent. Recently, Martelli et al. [18] found, for the same nozzle as $[\underline{7}, 8]$, that the self-sustained shock oscillations are mainly driven by a pressure imbalance between the pressure level downstream of the shock and the ambient flow. Based on the above discussion, it is clear that the flow separation in supersonic planar nozzles with unsteady flow conditions is still an active research topic where much effort is still needed to better understand the physical mechanism of the LFO. Intending to shed more light on this complex fluid problem, large-eddy simulations (LES), solving three-dimensional compressible Navier-Stokes equations with wall modeling approach, are used to study the flow separation in an overexpanded planar nozzle. The present paper is organized as follows. The next section introduces the governing equations followed by a complete description of both wallmodeling methodology and numerical methods. Then, the simulation setup is presented, followed by the results and discussion. Finally, conclusions are drawn at the end of the paper.

\section{Governing Equations, Turbulence Modeling, and Numerics}

The filtered compressible Navier-Stokes equations are written as

$$
\begin{gathered}
\partial_{t} \bar{\rho}+\partial_{j}\left(\bar{\rho} \widetilde{u}_{j}\right)=0 \\
\partial_{t}\left(\bar{\rho} \widetilde{u}_{i}\right)+\partial_{j}\left(\bar{\rho} \widetilde{u}_{i} \widetilde{u}_{j}\right)+\partial_{i} \bar{p}=\partial_{j} \breve{\sigma}_{i j}-\partial_{j} \tau_{i j} \\
\partial_{t}(\bar{\rho} \breve{E})+\partial_{j}\left(\bar{\rho} \breve{E} \widetilde{u}_{j}\right)+\partial_{i}\left(\widetilde{u}_{i} \bar{p}\right)=\partial_{j}\left(\breve{u}_{i} \sigma_{i j}\right)-\partial_{j} \breve{q}_{j}-\partial_{j}\left(\widetilde{u}_{i} \tau_{i j}\right)-\partial_{j} \mathcal{Q}_{j}
\end{gathered}
$$
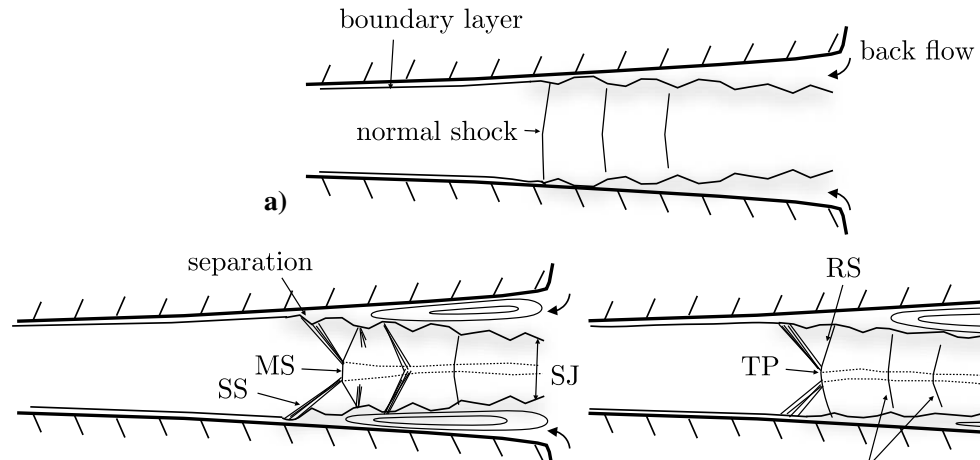

b)

recirculation bubbles

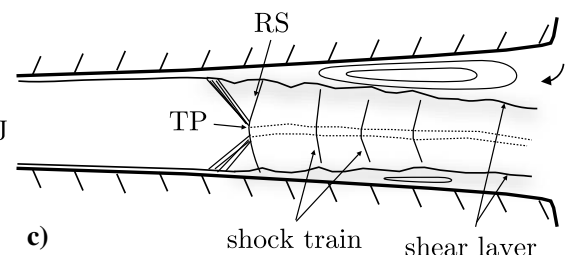

Fig. 1 Schematic representation of the typical flow regimes in a planar nozzle. MS: Mach stem; SS: separated shock; RS; reflected shock; TP: triple point; SJ: supersonic jet. 
where $\bar{\rho}, \widetilde{u}_{i}, \bar{p}$, and $\breve{T}$ are the filtered density, velocity, pressure, and temperature, respectively. Unlike the "bar" (Reynolds) and the "tilde" (Favre) filters, the "breve" symbol does not denote a filter operation but indicates that the quantity is based on primitive filtered variables. Thus, $\breve{E}$ refers to the resolved total energy, which is not equal to the filtered total energy. The resolved viscous stress tensor $\breve{\sigma}_{i j}$ and the heat flux $\breve{q}_{j}$ are defined as $\breve{\sigma}_{i j}=2 \mu(\breve{T})\left(\widetilde{S}_{i j}-\partial_{k} \widetilde{u}_{k} \delta_{i j} / 3\right)$ and $\breve{q}_{j}=-\lambda(\breve{T}) \partial_{j} \breve{T}$, respectively, where $\mu(\breve{T})$ and $\lambda(\breve{T})$ are the viscosity and the thermal conductivity corresponding to the filtered temperature $\breve{T}$ and $\widetilde{S}_{i j}=\left(\partial_{j} \widetilde{u}_{i}+\partial_{i} \widetilde{u}_{j}\right) / 2$. The low-pass filtering procedure generates several unclosed terms; most of these are neglected, except for the subgrid heat flux $\mathcal{Q}_{j}$ and the subgrid stress $\tau_{i j}$, which are modeled according to the eddy-viscosity hypothesis as

$$
\begin{gathered}
\mathcal{Q}_{j}=-k_{s g s} \partial_{j} \breve{T} \\
\tau_{i j}=-\mu_{s g s}\left(\partial_{j} \widetilde{u}_{i}+\partial_{i} \widetilde{u}_{j}-\frac{2}{3} \partial_{k} \widetilde{u}_{k} \delta_{i j}\right)+\frac{2}{3} k_{s g s} \delta_{i j} \\
\mu_{s g s}=C_{d} \Delta^{2} \bar{\rho}|\breve{S}|, \quad k_{s g s}=C_{I} \Delta^{2} \bar{\rho}|\breve{S}|^{2}, \quad \kappa_{s g s}=c_{p} \mu_{s g s} / P r_{s g s}
\end{gathered}
$$

where $c_{p}$ is the heat capacity at constant pressure and $k_{s g s}$ is the subgrid-scale kinetic energy. The modeling parameters $C_{d}, C_{I}$, and $P r_{s g s}$ are determined through the dynamic procedure of Lilly [19], Moin et al. [20], and Germano et al. [21] with filtering and averaging in the homogeneous (periodic) $z$ direction. $\Delta$ is the filter width associated with the wavelength of the smallest scale retained by the filtering operation.

\section{A. Near-Wall Turbulence Modeling}

The near-wall turbulence modeling is required in order to reduce the computational cost of fully resolving all turbulent scales in the boundary layer. Various strategies have been reported in the literature for the past decades, and many of those are case sensitive. The wallmodeling approach, adopted in this study, consists of using a regular LES grid near the wall combined with a wall model, to mimic the dynamical effects of the turbulent eddies in the near-wall layer. In this study, a thin-boundary-layer equations (TBLE) approach is adopted as in Kawai and Larsson [22]. The TBLE model of Kawai and Larsson [22] includes neither the pressure gradient nor the convective terms. This assumes that the boundary layer, below the exchange height of the wall model $\left(y_{w m}\right)$, is in equilibrium and by assuming that the pressure gradient from the LES domain is propagating onto the wall model through its top boundary condition. For the present study, a moderate pressure gradient is considered along the diverging section of the nozzle flow. Additionally, we have included a new formulation of the viscosity eddy, following the work of Duprat et al. [23], to partially account for the pressure gradient using a set of nondimensional variables derived from the work of Manhart et al. [24].

The final formulation of the TBLE model used in this study is as follows:

$$
\begin{gathered}
\partial_{t}\left(\bar{\rho} \widetilde{u}_{i}\right)+\partial_{j}\left(\bar{\rho} \widetilde{u_{i}} \widetilde{u_{j}}\right)=-\partial_{x} \bar{p}+\partial_{y}\left[\left(\mu+\mu_{t}\right) \partial_{y} \widetilde{u_{i}}\right] \\
\partial_{t}(\bar{\rho} \breve{E})+\partial_{x}\left(\bar{\rho} \breve{E} \widetilde{u_{j}}\right) \\
=-\widetilde{u}_{j} \partial_{x} \bar{p}+\partial_{y}\left[\left(\mu+\mu_{t}\right) \widetilde{u_{j}} \partial_{y} \widetilde{u_{j}}+c_{p}\left(\frac{\mu}{P_{r}}+\frac{\mu_{t}}{P_{r t}}\right) \partial_{y} \bar{T}\right]
\end{gathered}
$$

where $\mu_{t}, P_{r}$, and $P_{r t}$ are the eddy viscosity, the Prandtl number, and the turbulent Prandtl number, respectively. Following the work of Duprat et al. [23], the TBLE are reduced to the terms of the right-hand side. Under these assumptions, the simplified velocity streamwise momentum can be computed as follows:

$$
\partial_{y}(\widetilde{u})=\frac{\partial_{x}(\bar{p}) y+\tau_{w_{\mathrm{wm}}}}{\left(\mu+\mu_{t}\right)}
$$

where the wall-modeled shear stress $\tau_{w_{\mathrm{wm}}}$ and the turbulent viscosity $\mu_{t}$ are the unknown variables of the equation. Similarly, for adiabatic boundary conditions, the temperature can be computed using

$$
\partial_{y}(\bar{T})=\frac{\left(\mu+\mu_{t}\right) \widetilde{u} \partial_{y}(\widetilde{u})+\partial_{x}(\bar{p}) y \widetilde{u}}{c_{p}\left(\left(\mu / P_{r}\right)+\left(\mu_{t} / P_{r t}\right)\right)}
$$

The pressure gradient effect on the eddy viscosity is modeled by a nondimensional parameter $\alpha=u_{\tau}^{2} / u_{\tau, p}^{2}$, which quantifies the balance between the shear stress and the streamwise pressure gradient. The turbulent viscosity is defined by

$$
\mu_{t}=\mu \kappa y^{\star}\left[\alpha+y^{\star}(1-\alpha)^{3 / 2}\right]^{\gamma}\left(1-\exp \left(\frac{-y^{\star}}{\left(1+A \alpha^{3}\right)}\right)\right)^{2}
$$

where $\kappa$ is the von Kármán constant, $y^{\star}$ is the nondimensional length, and $A$ and $\gamma$ are the wall-model constants.

Dimensionless variables have been introduced to scale the boundary layer subjected to the pressure gradient, taking into account the wall-shear stress and the streamwise pressure gradient. The nondimensional velocity $u^{\star}$ and the length scale $y^{\star}$ are defined as $u^{\star}=\bar{u} / u_{\tau, p}$ and $y^{\star}=y u_{\tau, p} / \bar{\nu}$, where $u_{\tau, p}=\sqrt{\left(u_{\tau}^{2}+u_{p}^{2}\right)}$ is the combined velocity with $u_{\tau}=\sqrt{\tau_{w_{\mathrm{wm}}} / \bar{\rho}}$ the friction velocity, and $u_{p}=\left|\bar{\mu} / \bar{\rho}^{2} \partial_{x}(\bar{p})\right|^{1 / 3}$ the pressure-gradient-based velocity.

The wall model is characterized by an "input-output" exchange process, where the information extracted from the LES becomes the upper boundary condition for the TBLE model. The latter is then resolved numerically using the local one-dimensional grid of height $y_{\mathrm{wm}}$, to compute the wall-shear stress $\tau_{w_{\mathrm{wm}}}=\left(\mu \partial_{y} \widetilde{u}\right)_{y=0}$ and the heat flux $\dot{q}_{w_{\mathrm{wm}}}=-\left(\lambda \partial_{y} \bar{T}\right)_{y=0}$, which are injected back into the LES. Figure 2 shows a representation of the wall model methodology near the wall using the off-set method proposed by Kawai and Larsson [22].

The wall model has been implemented and assessed through a Direct Numerical Simulation (DNS) of a spatially evolving compressible boundary layer subjected to a pressure gradient. A comparison of the existing DNS of Pirozzoli et al. [25] has been first done to generate a developing boundary layer. The Reynolds number is taken as $R e_{x}=2.54 \times 10^{6}$, and the same perturbation model between $x=$ $4.5^{\prime \prime}$ to $x=5^{\prime \prime}$ is used as [25]. The inlet flow is set at Mach 2.25 and a static temperature of $16 \overline{9.4} 4 \mathrm{~K}$. The domain has been extended in the streamwise direction to have enough space to capture the separation/reattachment phenomena of the TBL. Then, the numerical data have been used to perform a DNS of a turbulent boundary layer under both adverse and favorable pressure gradients. Finally, the DNS of the nonequilibrium boundary layer was used to validate the correct implementation of the model through both a priori and a posteriori tests. The wall-model constants $A=17.2$ and $\gamma=0.78$ were determined through a priori tests (more details can be found in [26]).

\section{B. Numerical Flow Solver}

Numerical simulations are carried out using an in-house-validated compressible code. The convective terms are discretized using a
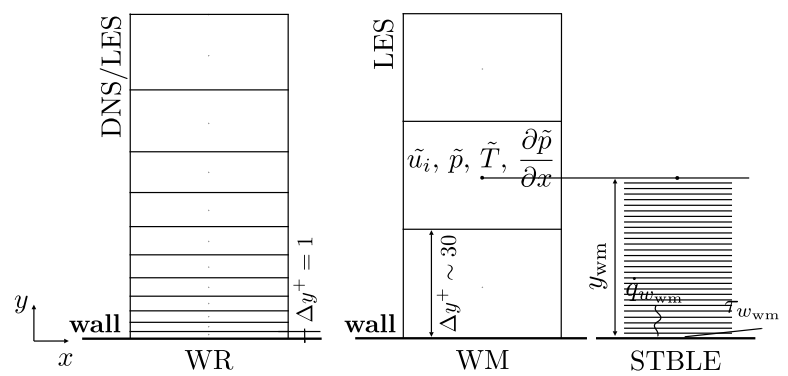

Fig. 2 Schematic representation of the wall-resolved and wall-modeled LES approaches. 


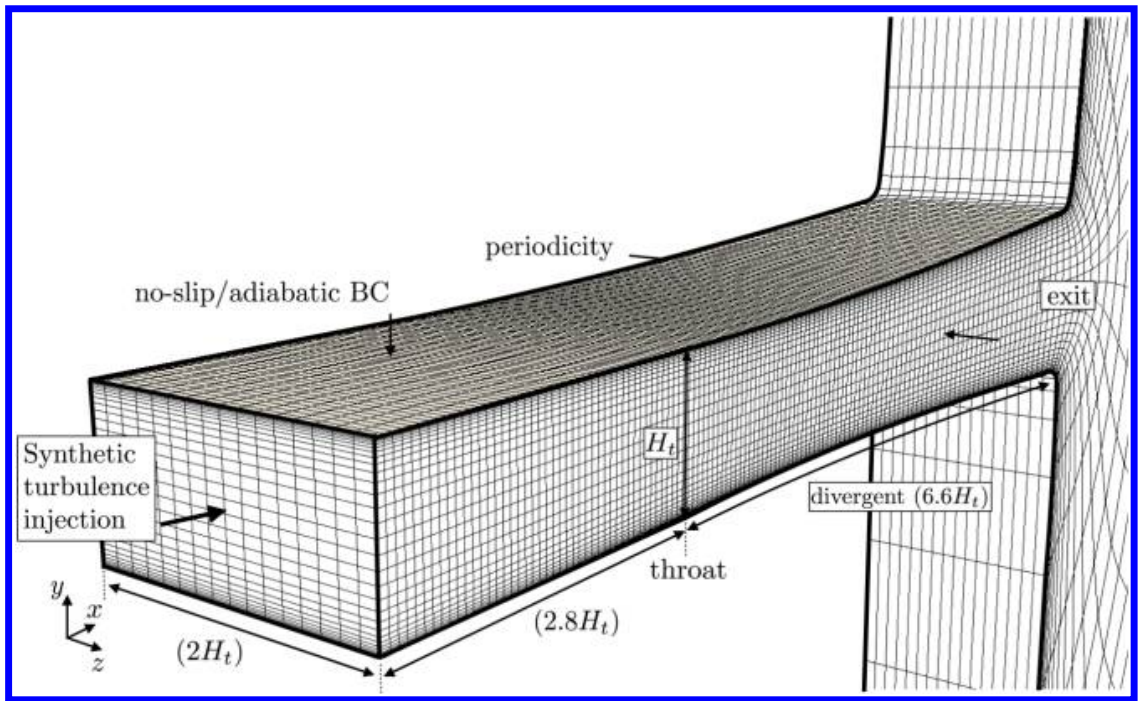

Fig. 3 Grid representation of the wall-resolved nozzle test case showing every 10th grid line. The grid stretching function is defined as $f_{\mathrm{str}, y}=\tanh \left(r_{v, y}\left(2 \eta / N_{y}-1\right)\right) / \tanh r_{v, y}$, where $N_{y}$ is the number of point and $\eta$ is the generalized curvilinear coordinate in wall-normal direction with $r_{v, y}=2.3$. For the WM-LES, the grid is regularly spaced following $f_{\text {str }, y}=\left(2 \eta / N_{y}-1\right)$. The nozzle throat is located at $x=0$.

sixth-order weighted compact nonlinear scheme (WCNS) developed by Deng and Zhang [27] in generalized curvilinear coordinates, whereas the viscous terms are discretized using a fourth-order central scheme with a split form of the Laplacian tensor for stability reason. The time is advanced using a fourth-order Runge-Kutta (RK4) scheme. The Courant-Friedrichs-Lewy (CFL) stability limit is near unity for all calculations. The solver is explicit and fully parallelized using MPI libraries.

\section{Problem Setup}

The test case considered in this study is the planar nozzle used in Olson and Lele [8], which is based on the experiments of Johnson and Papamoschou [7]. In the present simulations, the mesh is structured (see Fig. 3) and the incoming flow is generated using a synthetic turbulent boundary-layer inlet based on the method of Xie and Castro [28]. Similarly to [8] the converging part of the nozzle is not simulated here in order to reduce the computational cost of the overall simulations. To ensure a fully developed boundary layer at the nozzle throat, the computational inlet is shifted upstream using a buffer zone of length $L_{b}=2.8 H_{t}$, where $H_{t}$ is the throat height. The flow in the spanwise direction is assumed to be homogeneous with periodic boundary conditions $\left(L_{z}=2 H_{t}\right)$. The outlet boundary conditions are treated as nonreflective using the NSCBC method [29] in conjunction with an external buffer zone with a stretched grid to further damp the reflected waves.

Both upper and lower walls are considered as adiabatic with no-slip boundary conditions applied on the wall model. An NPR of 1.7 is considered, which corresponds to the experiments of Johnson and Papamoschou [7], with stronger shock amplitude and an asymmetric separation behavior. Olson and Lele [8] performed their LES for the same NPR and captured the same asymmetric separation behavior. The flow properties at the inlet (mainly the boundary-layer thickness $\left(\delta_{i}\right)$ and the freestream velocity $\left.\left(u_{i, \infty}\right)\right)$ are used as scaling parameters for $t^{\star}=t u_{i, \infty} / \delta_{i}$ and $S t_{\delta}=f \delta_{i} / u_{i, \infty}$. Although the current study is based on the use of a wall-modeled (WM-LES) approach, we have performed calculations using a wall-resolved (WR-LES) approach over a shorter period of time to ensure that the WM-LES restores the main trends of shock-boundary-layer interaction. Table 1 summarizes the grid resolutions used in both WR- and WM-LES compared with the one used by Olson and Lele []].

\section{Results and Discussion}

Mean flow quantities are collected from time averaging of the instantaneous three-dimensional fields that are extracted from a long-time series of the WM-LES data covering almost 70,000 characteristic time scales $\left(t^{\star}\right)$. This represents one of the longest time scale ever reached in separated nozzle flow simulations so far. Part of the WR-LES data is not shown here for concision. Figure 4 highlights an instantaneous picture of the complex three-dimensional nozzle flowfield showing the shock-wave-boundary-layer interaction pattern, with an asymmetric $\lambda$-shock structure. The whole shock pattern consists of an incident shock (IS), a reflected shock (RS), and a Mach stem (MS). In this configuration, the boundary-layer separation due to the high adverse pressure gradient creates an incident shock impinging the Mach stem at the triple-point TP. The reflected shock realigns the incident flow to the original direction. The Mach stem, which is a stronger shock, reduces the flow velocity substantially behind it to a subsonic state. The sonic line that bounds the shear layer and the recirculation zone can be seen as a virtual wall that defines the effective geometry of the nozzle. Because the effective nozzle geometry is shorter and has a lower expansion ratio, the resulting area reduction acts as an additional confinement effect of the flow, which compresses the flow and produces multiple shocks organized in a shock-train structure that is typically present in planar nozzles or transonic ducts.

\section{A. Mean Profiles}

The WR/WM LES pressure distribution at the wall is presented in Fig. 5a. The result agrees well with both experimental and previous LES results. The concordance between the data shows that the wall-modeled simulation is able to correctly reproduce the pressure gradient effect on the boundary layer. One can also notice a small difference in the separated region, where the experimental data and the WR computation show a smoother pressure jump compared with the wall-modeled simulation. This behavior is due to the strong compression waves in the wall-modeled simulation applying in the interaction zone upstream of the separation point.

Table 1 Grid parameters at the inlet of the nozzle

\begin{tabular}{lcccc}
\hline \hline & $\Delta x_{i}^{+}$ & $\Delta y_{i}^{+}$ & $\Delta z_{i}^{+}$ & Number of cells \\
\hline Olson and Lele [] & 30 & {$[1: 23]$} & 20 & $50.3 \mathrm{M}$ \\
WR-LES & 30 & {$[1: 29]$} & 20 & $73.4 \mathrm{M}$ \\
WM-LES & 60 & 45 & 40 & $13.7 \mathrm{M}$ \\
\hline \hline
\end{tabular}




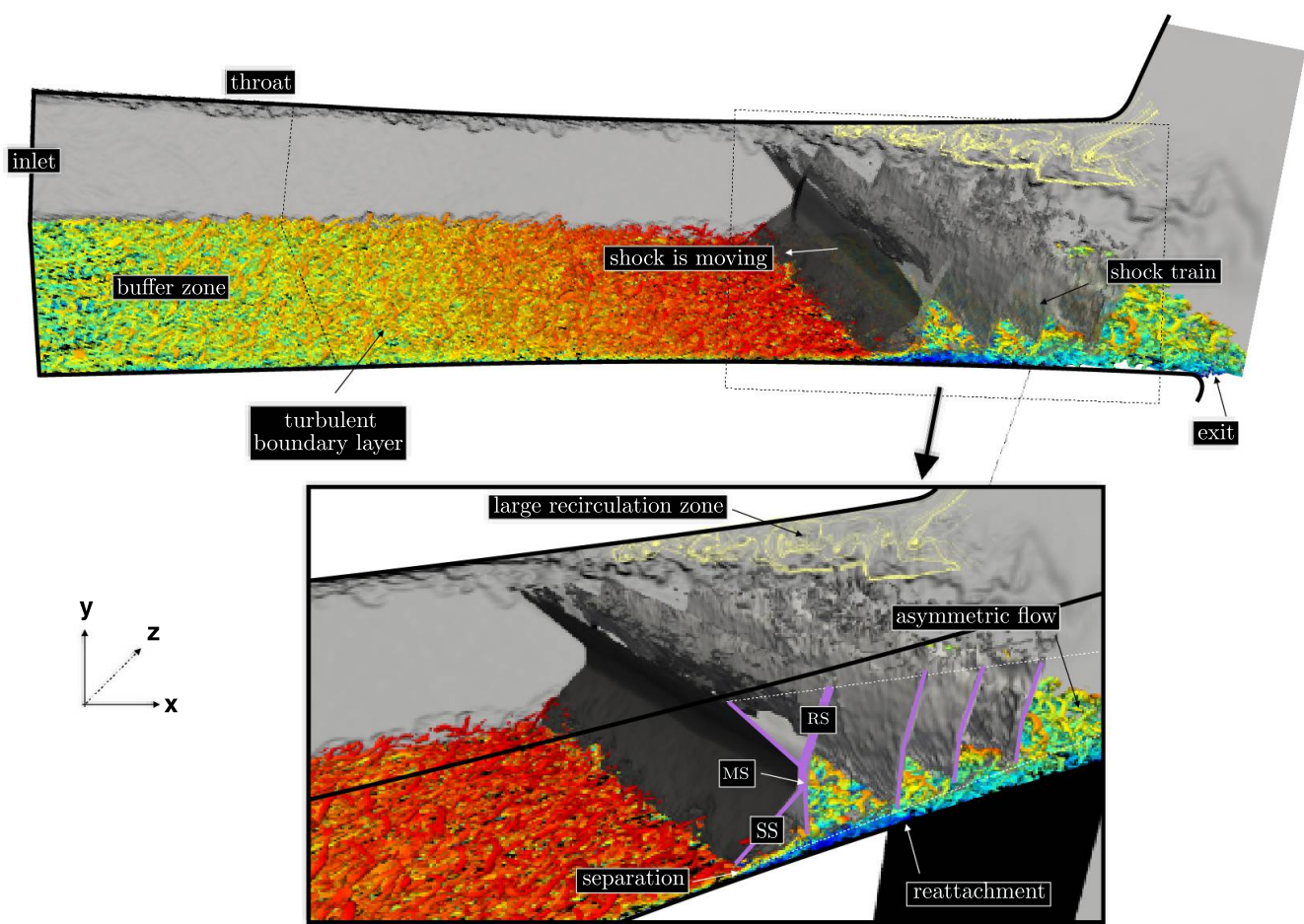

Fig. 4 Instantaneous three-dimensional nozzle flowfield showing isocontours of Q-criterion colored by the velocity field (blue: slow; red: fast). Grayscale surface represents schlieren imaging background in the $x$ - $y$ plane, whereas grayscale volume is the isovolume of the velocity gradient $\nabla\|u\|$. The image is taken when the separated shock was moving upstream toward the throat. See Fig. 1 for other notations.

The coarse grid of the wall-modeled case reduces the length of the interaction and increases the strength of the compression waves, resulting in a slightly stronger separated shock. Figure $5 \mathrm{~b}$ shows a comparison of the normalized pressure gradient using the Clauser's parameter $\beta=\delta^{\star} \partial_{x}(\bar{p}) / \tau_{w}$, where $\delta^{\star}=\delta / \delta_{i}$ is the normalized boundary-layer thickness. Upstream of the nozzle throat, the experimental data do not perfectly coincide with the simulations due to the difference in the converging part of the nozzle geometry. Note that the effect of the pressure gradient is accurately reproduced by the simulations, except in a region near the throat, where a small difference is noticeable.

\section{B. Shock Motion and Spectral Analysis}

To study the shock dynamics, one can track the separation position on both sides of the wall. The separation is found based on the location where the near-wall velocity becomes negative. Then, the noisy data are smoothed using a moving-average filter. The separation location is found to exhibit an oscillating behavior in a low-frequency range. Broadband and energetically significant LFO in the vicinity of the separated shock were also observed. The magnitude of the observed oscillations is close to the value found in [7], which is $8 \delta_{i}$. The flow separation at this particular NPR exhibits an asymmetric character with a jet flow being stuck on one side of the

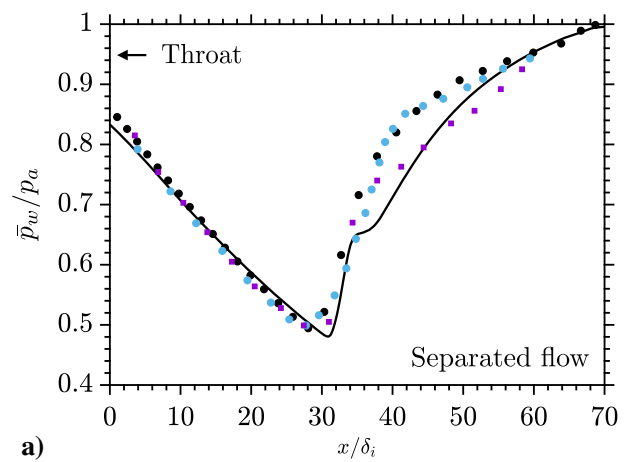

Fig. 5 a) Normalized mean wall-pressure distribution. b) Clauser's parameter along the longitudinal direction. —: WM-LES; •: WR-LES from [26]; •: WR-LES from []]; ஐ: experimental results from []]. nozzle without flipping, as reported in the experiments of Johnson and Papamoschou [7].

The mechanism of low-frequency unsteadiness in overexpanded nozzle flow has been described by Zaman et al. [15] in their experiments of transonic diffuser. They showed that the resonant tones occur when the length between the separation and the shock is similar to a quarter wavelength at the speed of sound $(c)$. This is true for small opening angles, transonic jet-Mach number and smoothnozzle walls. Zaman et al. [15] proposed the following correlation to compute the resonant tone, $\bar{f}_{r}=\left(c^{2}-u_{e}^{2}\right) / 4 c L_{s, e}$, where $L_{s, e}$ is the length between the averaged separation location and the exit of the nozzle, $c$ the speed of sound within the separated boundary layer, and $u_{e}$ the velocity at the exit.

Zaman's correlation provides a closer value to the resonant tone in comparison with the present WM-LES (i.e., $S t_{\delta} \sim 0.003$ ). In the present case, the jet Mach number $M_{j}$ is equal to 0.76 and the maximum half-angle of the nozzle is about $4^{\circ}$, which fits the transonic tone requirements. Similarly, Olson and Lele [8] concluded that the unsteady mechanism was due to a transonic resonant tone between the shock location and the nozzle exit. They found a twoway coupling between the shock and the shear layer combined with a time lag leading to the unsteady LFO. area $A_{e}$ and the velocity separation shock. It shows a time lag of

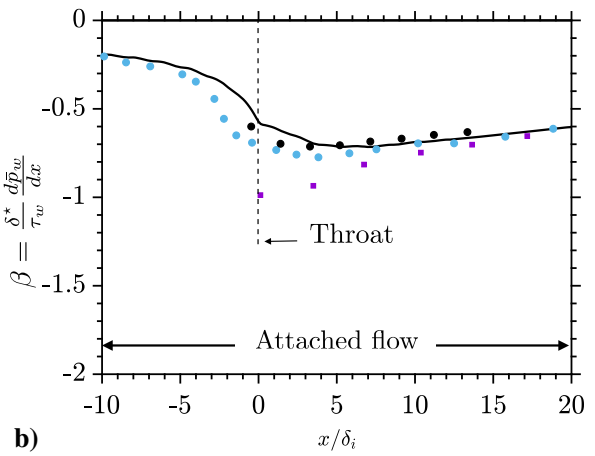

Figure $6 \mathrm{a}$ highlights the correlation between the effective exit 


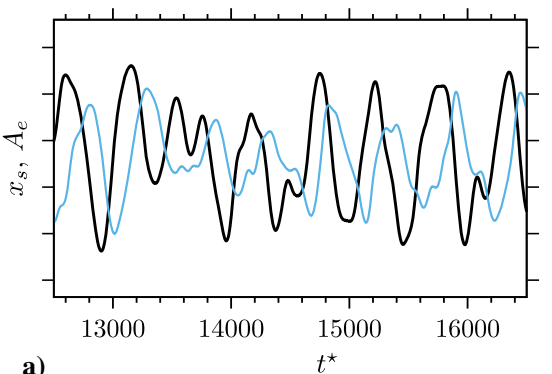

a)

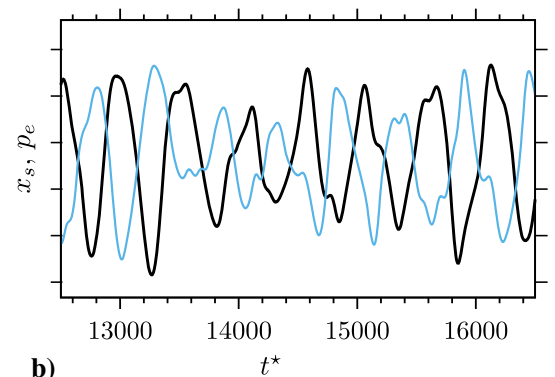

b)

Fig. 6 a) Time delay between the effective exit area $A_{e}(-)$ and the separation location $x_{s}(-)$. b) Time delay between the exit pressure $p_{e}(-)$ and the separation location $x_{s}(-)$.

approximately one-quarter wavelength, confirming the prediction of Olson and Lele [] w with respect to the resonant tone. Similarly, Fig. $\underline{6 b}$ shows the anticorrelation between the exit pressure $p_{e}$ and the separation location $x_{s}$. When the shock is located at its downstream position, the pressure is minimal because of the increased effective area. Conversely, when the shock is at its upstream position, the pressure is maximum considering the small effective area, which confirms the findings of Martelli et al. [18]. A small delay of $\Delta t^{\star} \sim$ 40 can be observed between the shock location and the exit pressure due to the acoustic waves that propagate upstream to the shock. In the present LES, the observed lag time between the shock-position signal and the effective-area signal was about one-quarter wavelength (i.e., $\Delta t^{\star} \sim 150$ ), confirming the resonance phenomenon observed by Zaman et al. [15]. This difference is not due to the time needed for the pressure waves from the confined exit area to propagate upstream of the shock (i.e., $\Delta t^{\star} \sim 40$ ), as addressed by Olson and Lele [ $\underline{8}$ ], but to the constant change of the effective area downstream of the shock.

The complex dynamics of the streamwise back and forth shock movement shown in Fig. 7 are summarized hereby, confirming previous scenarios of the following $[8,18]$ :

1) The separated shock moves downstream with a maximum shock speed $u_{s, \max }$. Because of the highly effective pressure at the nozzle exit resulting from the change of the effective geometry of the nozzle, the flow is then sucked up by the atmospheric pressure $p_{e}>p_{a}$. The relative speed of the incident shock is reduced because of the suction effects. At the end of this step, the incident shock vanishes to a series of compression waves that transform the $\lambda$-shock pattern into one normal shock.
2) At one-quarter wavelength, the exit pressure is lower than the ambient pressure, creating a slight backflow from the exit. This reversal flow starts to increase the deflection angle of the mixing layer and changes the effective geometry of the nozzle. The effective area starts to decrease with one-quarter delay compared with the position of the shock. Because of inertia (flow resistance), the shock is still moving toward the exit, whereas the backflow intensifies. At this stage, the flow is mildly symmetric and a backflow occurs on both sides of the nozzle.

3) When the shock is standing at its maximum position, the exit pressure is weak and the backflow from the ambient pressure increases. The mixing layer reattaches one of the nozzle walls (either upper or lower depending on the initial condition). The effective area is decreased compared with (2) and the flow is thermodynamically unbalanced, forcing thereby the shock position to adapt to the new state.

4) The shock moves upstream with a minimum shock speed $u_{s, \min }$. The mixing layer is fully attached to the lower wall, and the large recirculation bubbles emerge along the upper wall. The backflow acts only on the upper wall, which is opened to the atmosphere. The effective geometry of the nozzle downstream of the shock behaves as a convergent nozzle, where the subsonic flow accelerates to a supersonic stream and forms a series of shocks known as "shock train."

5) After five quarters of wavelength, the shock is still moving upstream but the exit pressure becomes higher than the atmospheric one. As for the one quarter wavelength time step, the effective crosssectional area of the nozzle starts to increase, which unbalanced the total pressure at the exit.

6) The shock is now at its most upstream location within the cycle. The suction effect from the ambient pressure forces the mixing

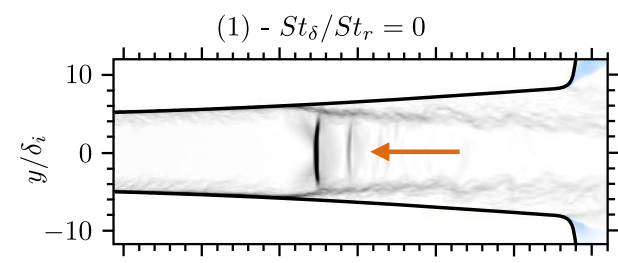

(3) $-S t_{\delta} / S t_{r}=\pi / 2$

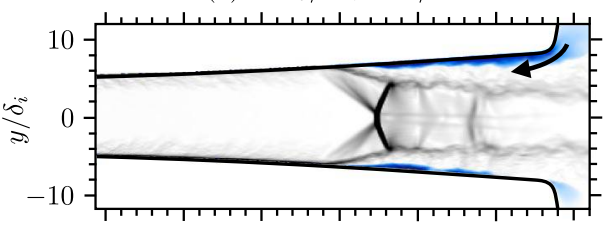

(5) $-S t_{\delta} / S t_{r}=5 \pi / 4$

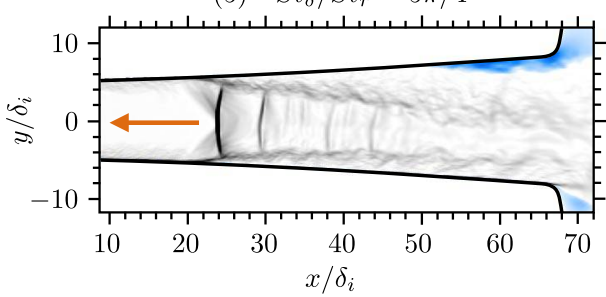

(2) $-S t_{\delta} / S t_{r}=\pi / 4$

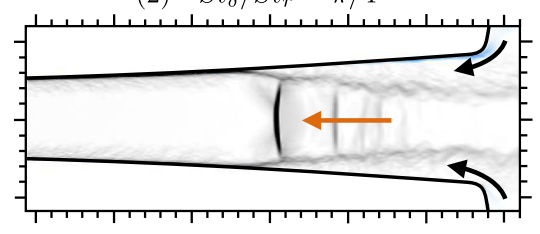

(4) $-S t_{\delta} / S t_{r}=\pi$

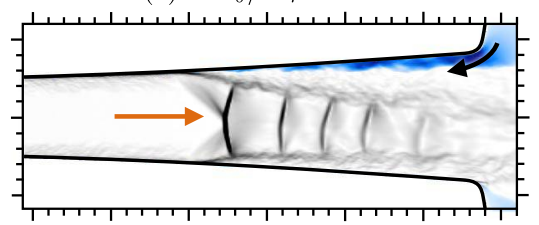

(6) $-S t_{\delta} / S t_{r}=3 \pi / 2$

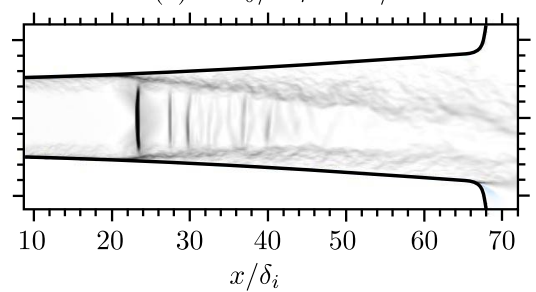

Fig. 7 Shock excursion over one resonant tone period $S t_{r}$ for different time steps. $z$-averaged schlieren pictures shown in grayscale, blue regions highlight negative $x$ velocity, orange arrows indicate the direction of the shock movement, and black arrows indicate the backflow. 


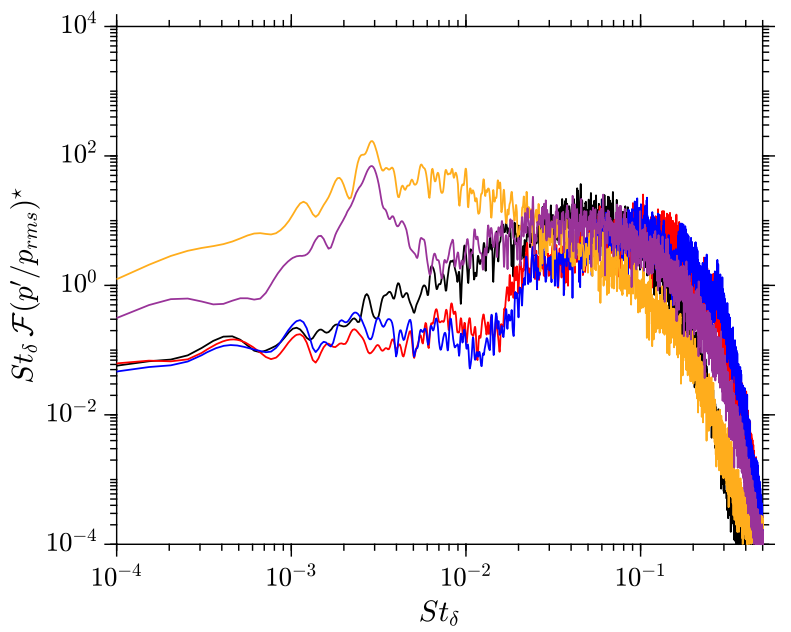

Fig. 8 Premultiplied spectrum of the normalized wall-pressure fluctuations for different streamwise locations. - : inlet; -: $x / \delta_{i}=-12$ upstream of the throat; - : throat region; - : separation region; recirculation zone.

layer to move closer to the walls, increasing the effective area. As for (3), the flow is thermodynamically unbalanced, forcing the shock position to move downstream. The acoustic lag due to the time taken for the pressure waves to acoustically propagate from the back pressure to the shock is maximal at this position, increasing thereby the unsteadiness of the shock.

Hence, a restart cycle from (1) maintains the shock in a closed-loop motion.

Figure 8 shows the normalized premultiplied spectra of pressure probe signals in five different locations. The spectra, defined as $\operatorname{St} \mathcal{F}\left(p^{\prime} / p_{\text {rms }}\right)^{\star}=S t \mathcal{F}\left(p^{\prime} / p_{\text {rms }}\right) / \int_{S t} S t \mathcal{F}\left(p^{\prime} / p_{\text {rms }}\right) \mathrm{d} S t$, cover the entire range of frequencies, from very low up to the boundary-layer time scales (i.e., $S t_{\delta}>1$ ). The spectrum at the throat shows no

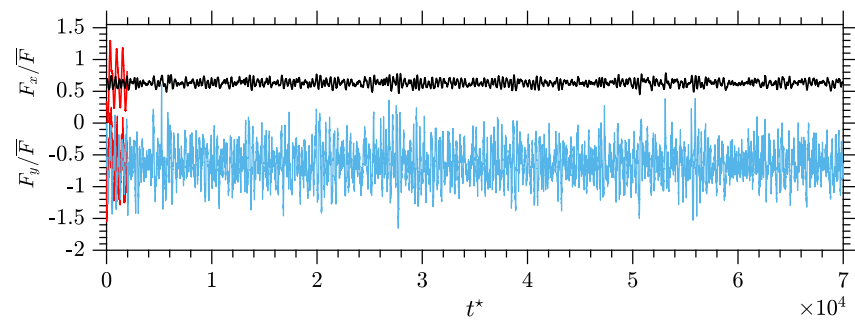

Fig. 9 a) Instantaneous wall-pressure forces integrated along the upper and the lower walls. Data have been filtered with a cutoff Strouhal number of 0.2. —: streamwise forces; - : side loads; - WR-LES from Piquet [26]. low-frequency activities. The same remark holds for the probe at the inlet and at $x / \delta_{i}=-12$ upstream of the throat. However, the main tone is captured both at the separation location and at the nozzle exit. In the recirculation zone, most of the spectral energy is contained in a region where $S t_{\delta} \sim 0.04$ as observed by [30]. They showed that the recirculating area exhibits large oscillations in the low-frequency range. Most of the energy captured in the recirculating area is due to the breathing phenomenon caused by an imbalance state between the flow from the shear layer and the backflow near the nozzle lip. The corresponding main tone is captured at the nozzle exit, where both recirculation bubble and lip vortex phenomenon interact. The later, having a small size compared with the main recirculation zone, is created by the confluence of the recirculation zone and the backflow.

Figure $\underline{9}$ shows the time-history of the WM-LES-integrated wall-pressure force compared with the WR-LES results of Piquet [26]. It is worth mentioning that the WM-LES integration time represents 18 times the one found in Piquet [26], Olson and Lele [8], and the experiments in [7]. Thus, the present integrated time is large enough to resolve several shock excursions, therefore having better statistics convergence.

The wall-pressure vector force is integrated along both upper and lower walls as $\boldsymbol{F}=\int_{s}\left(p_{w}-p_{a}\right) \boldsymbol{n} \mathrm{d} s$, where $p_{w}$ is the wall pressure, $p_{a}$ the ambient pressure, $d s$ the local surface element where the force is applied, $\boldsymbol{n}$ the normal vector to the surface, and $S$ the total surface of the nozzle including the upper and lower wall. The forces are decomposed into streamwise and lateral forces (side loads).

Figure 10 shows the normalized premultiplied spectra of the normalized streamwise/side-loads force fluctuations $\operatorname{St} \mathcal{F}\left(F_{i}^{\prime} /\langle F\rangle\right)^{\star}=$ $S t \mathcal{F}\left(F_{i}^{\prime} /\langle F\rangle\right) / \int_{S t} S t \mathcal{F}\left(F_{i}^{\prime} /\langle F\rangle\right) \mathrm{d} S t$, compared with the results of [26]. One can clearly notice that the two spectra are quietly converged for the high frequencies, while the main tone is different. This difference is due to the shock position in both simulations as a direct result of using the NSBC conditions at the outlet in the present simulation, which prevents the reflected acoustic wave from affecting the shock system and causing large-shock oscillations. Figure 10 shows that the streamwise forces fluctuate at a low frequency, whereas the side loads contain a broad range of frequency unsteadiness. In the streamwise force spectrum, three peaks can be observed at $S t_{\delta} \sim 0.003, S t_{\delta} \sim 0.04$, and $S t_{\delta} \sim 0.1$, corresponding to the resonant tone, the recirculation bubble, and the mixing layer phenomenon, respectively. High-frequency peaks can be observed in the side-loads spectrum at $S t_{\delta} \sim 0.04$ and $S t_{\delta} \sim 0.15$. It seems that the side loads are highly sensitive to the high-frequency oscillations, whereas the streamwise force is more sensitive to the main tone of the separation line.

Each phenomenon occurring in the nozzle can be characterized by a frequency range. To identify these frequencies (Fig. 10) and to link them to the corresponding physical phenomena, a dynamic mode decomposition (DMD) analysis is performed. Given the wide scale of frequencies involved in this flow and in order to extract the
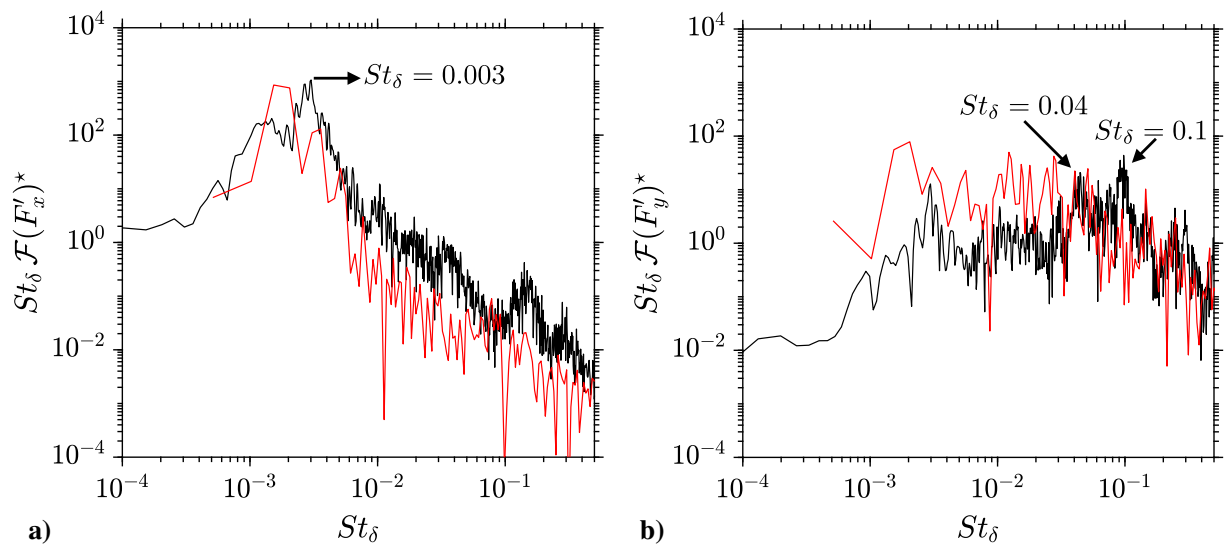

Fig. 10 Normalized premultiplied spectra of dimensionless fluctuations: a) streamwise forces; b) side loads. —: WR-LES from Piquet [26]; —: present WM-LES. 

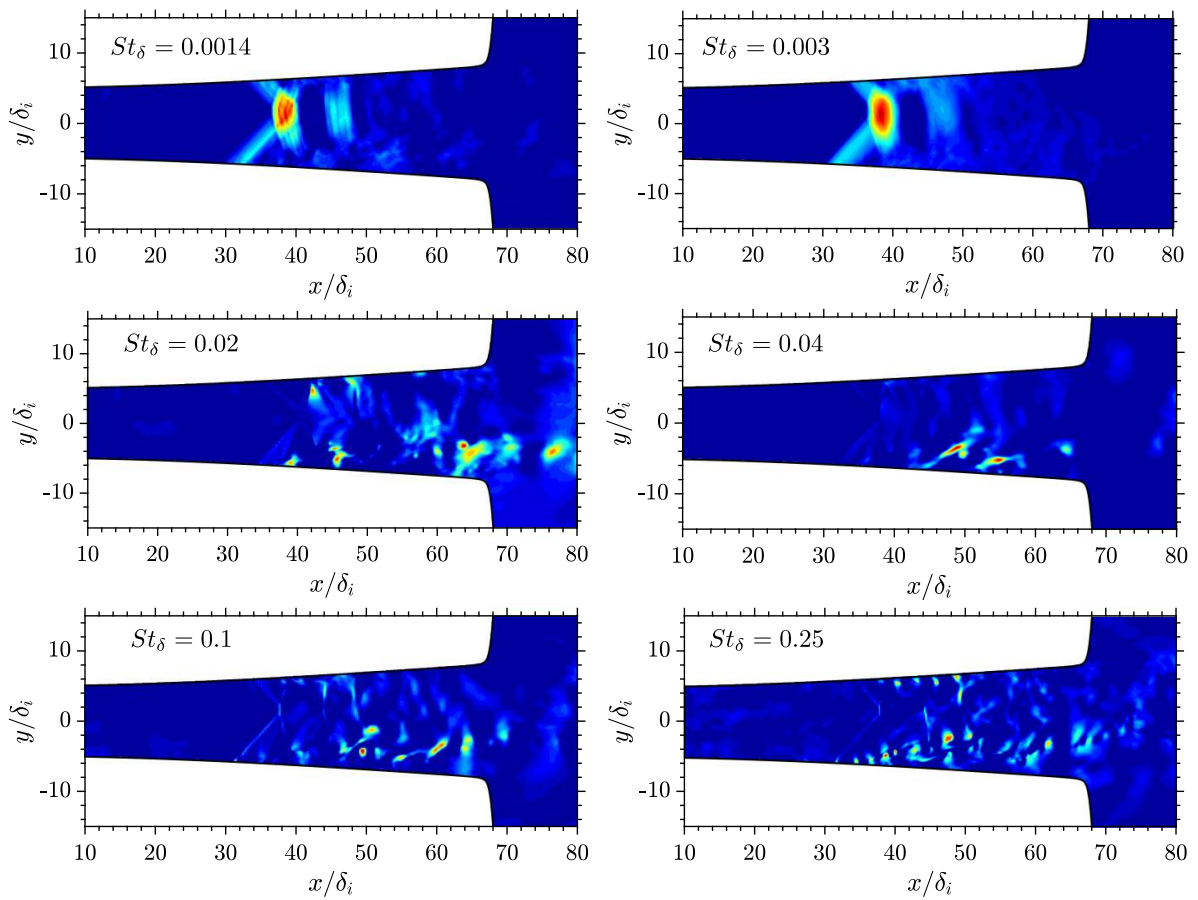

Fig. 11 Reconstructed field of the real part of the pressure fluctuations, $p^{\prime}$, for different DMD modes at selected values of $S t_{\delta}$. Red is the highest representative phenomenon of the mode.

corresponding low frequencies, we have used a multiresolution DMD (MrDMD) developed by Kutz et al. [31]. The MrDMD is able to separate robustly the complex systems into a hierarchy of multiresolution time-scale components. The method is applied to a series of two-dimensional slices of pressure fluctuations $p^{\prime}$, where each snapshot represents the full flowfield (the nozzle flow and the ambient region). Equi-time spaced snapshots are used and sampled at $S t_{\delta}=1$, which gives a Nyquist frequency of $S t_{\delta}=0.5$. Thus, the entire frequency range can be captured. The results shown in Fig. 11 are summarized as follows:

1) $S t_{\delta} \in[\ldots, 0.009]$; a frequency range corresponds to the oscillation of the shock system. Note that the resonant tone predicted by Zaman et al. [15] is captured at the separation and downstream at $S t=0.003$.

2) $S t_{\delta} \in[0.01,0.09]$; the subsonic flow downstream of the separation is trapped between the mixing layer and the wall, producing small unsteady eddies that oscillate within this range of frequencies (recirculation bubbles). This phenomenon is strongly affecting the side loads.

3) $S t_{\delta} \in[0.1,0.5]$; the mixing layer developing from the boundary-layer separation to the nozzle exit is observed at these frequencies. The large two-dimensional Kelvin-Helmholtz vortices impact the exit conditions, modifying thereby the effective nozzle exit area and the corresponding shock location.

4) $S t_{\delta} \sim 1$; upstream of the separation, the pressure fluctuations are dominant in the range of the turbulent boundary-layer frequencies.

\section{Conclusions}

A wall-modeled LES approach is used to investigate the unsteady behavior of three-dimensional flow separation in a supersonic planar nozzle. The objective of the study is to provide a better understanding of the main mechanisms driving the shock oscillations. The absence of low-frequency phenomena upstream of the boundary-layer separation and their presence near the separation point and in the downstream region confirm the role of the downstream flow conditions in driving or at least in maintaining the shock oscillations at very low frequencies. A scenario describing the way in which the LFO occur is proposed confirming the earlier findings. The study shows that the deflection angle of the separated shear layer, which represents the effective geometry of the nozzle downstream of the shock, is strongly coupled to the shock position. A phase shift between the motion of these two features, that is, upstream or downstream movement, causes the cycle to constantly overcompensate its pressure to match the exit pressure, giving thereby the self-sustained shock motion behavior already observed in many experimental and numerical setups. Regarding the asymmetry behavior of the separation, the current simulations show that the phenomenon is stable for the studied NPR and does not switch from asymmetric to symmetric flow separation over a very long run time, confirming the experimental findings. This confirmation was possible thanks to the use of the current wall model strategy, which allows, by its ability to correctly model the turbulent boundary layer and consequently the reduction of the number of mesh points, to have very long signals required for the study of the LFO.
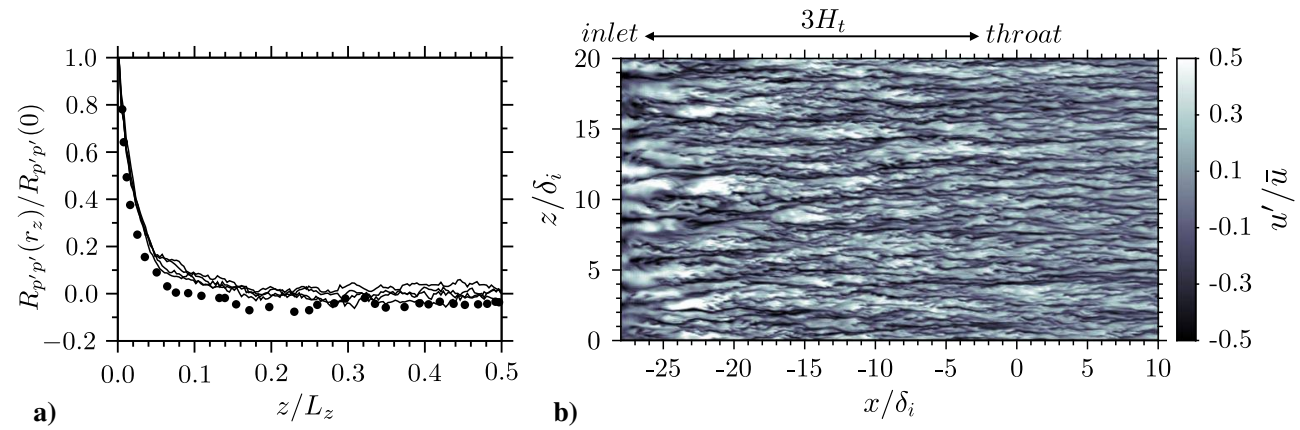

Fig. A1 a) Two-point correlation function of wall pressure fluctuations along the spanwise direction $\left(L_{z}=2 H_{t}\right)$ at $x / \delta_{i}=0($ - $:$ results at different $x$ locations in the preshock boundary-layer zone; ๑: WR-LES from [8]). b) Instantaneous velocity fluctuations in the $x-z$ plane at $y^{+} \sim 20$. 


\section{Appendix A: Grid Sensitivity Study}

\section{A.1. Periodic Domain Extent and Boundary-Layer Development}

To ensure that the domain size in the $z$ direction is large enough to cover most of the length scales present in the flow, a two-point correlation function based on the wall pressure along the spanwise direction, $\quad R_{p^{\prime} p^{\prime}}\left(r_{z}\right)=\sum_{n=1}^{N_{z}} \overline{p_{n}^{\prime} p_{n+n_{r}}^{\prime}}$, with $n_{r}=0, \ldots, N_{z}$; $r_{z}=n_{r} \Delta z$, is computed and averaged over time for two $x$ locations. Figure A1a shows $R_{p^{\prime} p^{\prime}}\left(r_{z}\right) / R_{p^{\prime} p^{\prime}}(0)$ plotted at the throat. The decay of correlation at $z / L_{z}=0.1$ demonstrates that the length in the spanwise direction does not affect the dynamics and the development of the coherent structure in the flow.

For the development of the boundary layer, the synthetic turbulence injection starts at $x / \delta_{i}=-28$, giving 18 boundary-layer thicknesses length for the flow to relax to a converged boundary layer. Xie and Castro [28] have demonstrated that the method of synthetic injection needs at least 10 boundary-layer thicknesses to reach a fully turbulent flow. Figure A1b shows the structure of the turbulence in the boundary layer at the buffer layer through the velocity fluctuations field. The synthetic eddy structures seem to extend to $\Delta x / \delta_{i} \sim 15$, in agreement with the streaky structures as observed by Xie and Castro [28]. Past this recovery region, the velocity fluctuations exhibit more coherent structures.

\section{A.2. Mesh Grid Convergence}

To establish the fidelity of the LES database, we conducted an LES grid convergence study. Three different meshes are used (see Table A1). The normal direction has been excluded from this study because the Kawai and Larsson's [22] recommendations are strictly followed and we believe that it is sufficient for the convergence in the wall-normal direction. Thus, the methodology consists of changing the number of points in both streamwise and spanwise directions. The obtained results are presented in Fig. A2b in terms of normalized mean wall pressure distribution for different grid

Table A1 LES grid parameters

\begin{tabular}{lcccc}
\hline \hline & $N_{x}$ & $N_{y}$ & $N_{z}$ & Number of cells \\
\hline Mesh A & 672 & 128 & 160 & $13.7 \mathrm{M}$ \\
Mesh B & 750 & 128 & 160 & $15.4 \mathrm{M}$ \\
Mesh C & 672 & 128 & 190 & $18.3 \mathrm{M}$ \\
\hline \hline
\end{tabular}

Table A2 Wall model grid parameters

\begin{tabular}{lcc}
\hline \hline & Exchanging cell & $N_{w m}$ \\
\hline Case 1 & 2 & 50 \\
Case 2 & 3 & 50 \\
Case 3 & 3 & 100 \\
\hline \hline
\end{tabular}

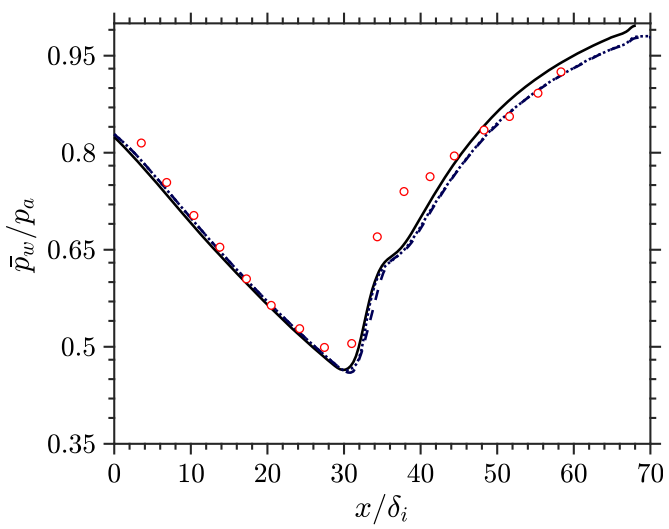

Fig. A3 Normalized mean wall-pressure distribution (一: case 1; ---: case 2; $\circ$ : case 3). See Table A2.

resolutions. Although the statistics were not acquired over the same amount of samples, the number of samples used in this study was large enough to consider the results as statistically converged. From Fig. A2b, it is seen that varying the grid resolution does not produce significant differences in the wall pressure before the separation and in the shocked region. Therefore, we consider that the grid resolution, used in this study, is sufficiently fine to only have marginal effects on the statistical results.

Figure 2 shows schematically the concept of WM-LES where the TBLE equations are solved on a separate grid. In the present simulation, the exchange of information between the LES and the wall model occurs at $y_{w m}$, corresponding to the second LES cell away from the wall. Kawai and Larsson [22] recommended to avoid the log-layer mismatch of the WM-LES when the exchange occurs in the first or in the second cell due to numerical errors in the LES solution at those two first cells. Hence, we have considered adding three cells below. The results are shown in Fig. A3. It is clear that adding one more cell leads to a small underprediction of the wall pressure. Note that all recommendations made by Kawai and Larsson [22] are respected, expect using four or five cells below the exchanging height.

\section{Appendix B: Wall-Model Sensitivity Study}

Several authors have shown that the error incurred by neglecting both pressure gradient and convective terms in the TBLE equations can be larger than the error incurred by an equilibrium formulation, that is, not accounting for those two terms in the wall model. For this purpose, a new simulation has been performed using the present wall model without the pressure gradient term (PGT). The wall pressure from the new simulation, together with the previous data and the experiment results from [7], is presented in Fig. B1. Indeed, a quite small improvement is noticed in the recovery region downstream of the separated zone when the pressure gradient term is included, whereas the separation location remains the same in both formulations.
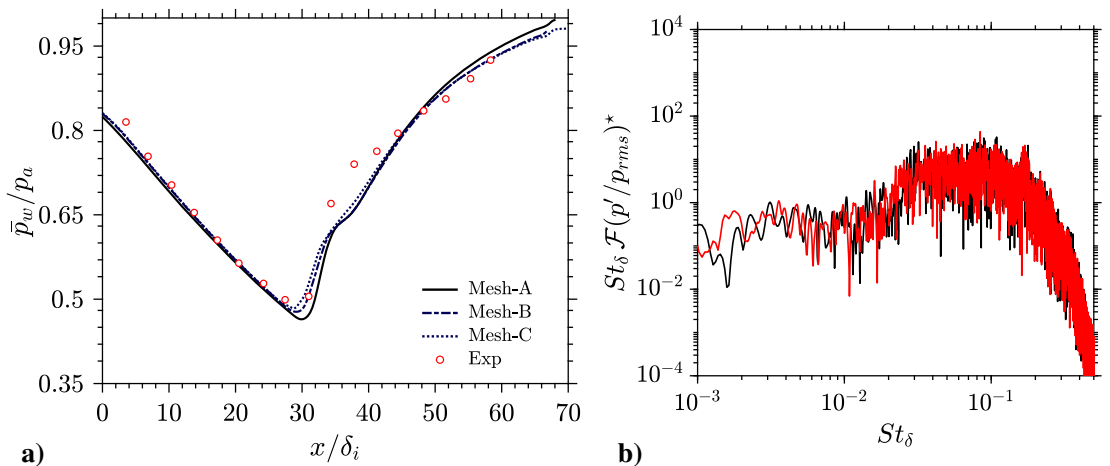

Fig. A2 a) Normalized mean wall-pressure distributions for different meshes. b) Normalized premultiplied spectra of the normalized pressure fluctuations at the throat (一: mesh $\mathrm{A}$; -: mesh $\mathrm{B}$. 


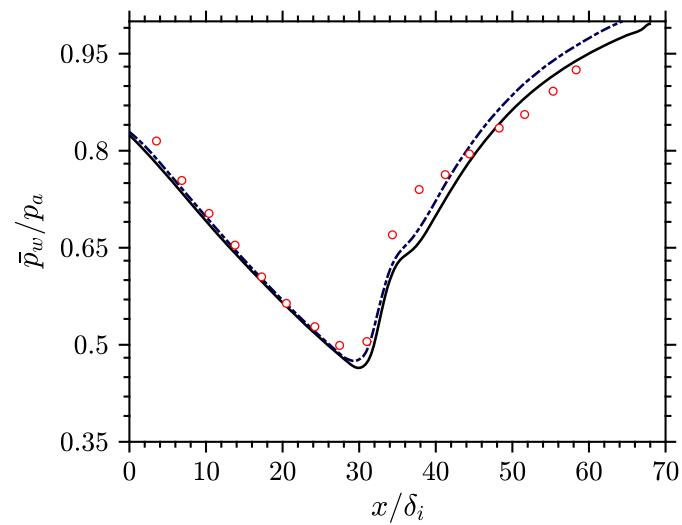

Fig. B1 Normalized mean wall-pressure distribution. -: WM-LES with PGT; - - -: WM-LES without PGT; $\odot$ : experimental results from [7].

\section{Acknowledgments}

B.Z. gratefully acknowledges support from the Algerian Government through a Ph.D. fellowship. The authors thank Emile Touber from Imperial College London for his valuable discussion on the preliminary version of this work. The use of high performance computing resources from Centre Régional Informatique et d'Applications Numériques de Normandie (CRIANN), Rouen, France, under the allocation 1998022, is acknowledged.

\section{References}

-11] Hadjadj, A., and Onofri, M., "Nozzle Flow Separation," Shock Waves, Vol. 19, No. 3, 2009, pp. 163-169. https://doi.org/10.1007/s00193-009-0209-7

[2] Papamoschou, D., and Johnson, A., "Unsteady Phenomena in Supersonic Nozzle Flow Separation,” AIAA Paper 2006-3360, 2006.

-[3] Xiao, Q., Tsai, H. M., and Papamoschou, D., "Numerical Investigation of Supersonic Nozzle Flow Separation," AIAA Journal, Vol. 45, No. 3 , 2007, pp. 532-541. https://doi.org/10.2514/1.20073

- [4] Hunter, C. A., "Experimental Investigation of Separated Nozzle Flows," Journal of Propulsion \& Power, Vol. 20, No. 3, 2004, pp. 527-532. https://doi.org/10.2514/1.4612

[5] Verma, S., and Manisankar, C., "Origin of Flow Asymmetry in Planar Nozzles with Separation," Shock Waves, Vol. 24, No. 2, 2014, pp. 191-209. https://doi.org/10.1007/s00193-013-0492-1

[6] Bourgoing, A., and Reijasse, P., "Experimental Analysis of Unsteady Separated Flows in a Supersonic Planar Nozzle," Shock Waves, Vol. 14, No. 4, 2005, pp. 251-258. https://doi.org/10.1007/s00193-005-0269-2

-[7] Johnson, A. D., and Papamoschou, D., "Instability of Shock-Induced Nozzle Flow Separation," Physics of Fluids, Vol. 22, No. 1, 2010, Paper 016102. https://doi.org/10.1063/1.3278523

[8] Olson, B. J., and Lele, S. K., "Large-Eddy Simulation of an Over-Expanded Planar Nozzle,” AIAA Paper 2011-3908, 2011.

-[9] Verma, S., Chidambaranathan, M., and Hadjadj, A., "Analysis of Shock Unsteadiness in a Supersonic Over-Expanded Planar Nozzle," European Journal of Mechanics-B/Fluids, Vol. 68, March-April 2018, pp. 55-65. https://doi.org/10.1016/j.euromechflu.2017.11.005

[10] Dussauge, J. P., Dupont, P., and Debiève, J. F., "Unsteadiness in Shock Wave Boundary Layer Interaction with Separation," Aerospace Science and Technology, Vol. 10, No. 2, 2006, pp. 85-91. https://doi.org/10.1016/j.ast.2005.09.006

[11] Dupont, P., Haddad, C., Ardissone, J. P., and Debiève, J. F., "Space and Time Organisation of a Shock Wave/Turbulent Boundary Layer Interaction," Aerospace Science and Technology, Vol. 9, No. 7, 2005, pp. 561-572. https://doi.org/10.1016/j.ast.2004.12.009

[12] Shimshi, E., Ben-Dor, G., Levy, A., and Krothapalli, A., "Asymmetric and Unsteady Flow Separation in High Mach Number Planar Nozzles," International Journal of Aeronautical Science \& Aerospace Research, Vol. 2, No. 6, 2015, pp. 65-80. https://doi.org/10.19070/2470-4415-150008
[13] Chaudhuri, A., and Hadjadj, A., "Numerical Investigations of Transient Nozzle Flow Separation,” Aerospace Science and Technology, Vol. 53, June 2016, pp. 10-21. https://doi.org/10.1016/j.ast.2016.03.006

[14] Ganapathisubramani, B., Clemens, N. T., and Dolling, D. S., "Effects of Upstream Boundary Layer on the Unsteadiness of Shock-Induced Separation," Journal of Fluid Mechanics, Vol. 585, Aug. 2007, pp. 369-394. https://doi.org/10.1017/S0022112007006799

[15] Zaman, K., Dahl, M. D., Bencic, T. J., and Loh, C. Y., "Investigation of a Transonic Resonance with Convergent-Divergent Nozzles," Journal of Fluid Mechanics, Vol. 463, July 2002, pp. 313-343. https://doi.org/10.1017/S0022112002008819

[16] Touber, E., and Sandham, N. D., "Large-Eddy Simulation of Low-Frequency Unsteadiness in a Turbulent Shock-Induced Separation Bubble," Theoretical and Computational Fluid Dynamics, Vol. 23, No. 2, 2009, pp. 79-107. https://doi.org/10.1007/s00162-009-0103-z

[17] Bruce, P. J. K., and Babinsky, H., "Unsteady Shock Wave Dynamics," Journal of Fluid Mechanics, Vol. 603, May 2008, pp. 463-473. https://doi.org/10.1017/S0022112008001195

[18] Martelli, E., Ciottoli, P. P., Saccoccio, L., Nasuti, F., Valorani, M., and Bernardini, M., "Characterization of Unsteadiness in an Overexpanded Planar Nozzle," AIAA Journal, Vol. 57, No. 1, 2019, pp. 239-251. https://doi.org/10.2514/1.J057162

[19] Lilly, D. K., "A Proposed Modification of the Germano Subgrid-Scale Closure Method," Physics of Fluids, Vol. A4, No. 3, 1992, pp. 633-635. https://doi.org/10.1063/1.858280

[20] Moin, P., Squires, K., Cabot, W., and Lee, S., "A Dynamic Subgrid-Scale Model for Compressible Turbulence and Scalar Transport," Physics of Fluids, Vol. 3, No. 11, 1991, pp. 2746-2757. https://doi.org/10.1063/1.858164

[21] Germano, M., Piomelli, U., Moin, P., and Cabot, W. H., "A Dynamic Subgrid-Scale Eddy Viscosity Model,” Physics of Fluids, Vol. 3, No. 7, 1991, pp. 1760-1765. https://doi.org/10.1063/1.857955

[22] Kawai, S., and Larsson, J., "Wall-Modeling in Large Eddy Simulation: Length Scales, Grid Resolution, and Accuracy," Physics of Fluids, Vol. 24, No. 1, 2012, Paper 015105. https://doi.org/10.1063/1.3678331

[23] Duprat, C., Balarac, G., Métais, O., Congedo, P. M., and Brugière, O., "A Wall-Layer Model for Large-Eddy Simulations of Turbulent Flows with/out Pressure Gradient," Physics of Fluids, Vol. 23, No. 1, 2011, Paper 015101 https://doi.org/10.1063/1.3529358

[24] Manhart, M., Peller, N., and Brun, C., "Near-Wall Scaling for Turbulent Boundary Layers with Adverse Pressure Gradient," Theoretical and Computational Fluid Dynamics, Vol. 22, Nos. 3-4, 2008, pp. 243-260. https://doi.org/10.1007/s00162-007-0055-0

- [25] Pirozzoli, S., Grasso, F., and Gatski, T. B., "Direct Numerical Simulation and Analysis of a Spatially Evolving Supersonic Turbulent Boundary Layer at M = 2.25," Physics of Fluids, Vol. 16, No. 3, 2004, pp. 530-545. https://doi.org/10.1063/1.1637604

[26] Piquet, A., "Physical Analysis and Numerical Simulation of the Separation Phenomenon in Over-Expanded Nozzle Flow," Ph.D. Thesis, Normandie Université, Rouen, France, 2017.

[27] Deng, X., and Zhang, H., "Developing High-Order Weighted Compact Nonlinear Schemes," Journal of Computational Physics, Vol. 165, No. 1, 2006, pp. 22-44. https://doi.org/10.1006/jcph.2000.6594

[28] Xie, Z. T., and Castro, I. P., "Efficient Generation of Inflow Conditions for Large Eddy Simulation of Street-Scale Flows," Turbulence \& Combustion, Vol. 81, No. 3, 2008, pp. 449-470. https://doi.org/10.1007/s10494-008-9151-5

[29] Poinsot, T. J., and Lele, S., "Boundary Conditions for Direct Simulations of Compressible Viscous Flows," Journal of Computational Physics, Vol. 101, No. 1, 1992, pp. 104-129. https://doi.org/10.1016/0021-9991(92)90046-2

[30] Simon, F., Deck, S., Guillen, P., Sagaut, P., and Merlen, A., "Numerical Simulation of the Compressible Mixing Layer Past an Axisymmetric Trailing Edge," Journal of Fluid Mechanics, Vol. 591, Nov. 2007, pp. $215-253$. https://doi.org/10.1017/S0022112007008129

[31] Kutz, J. N., Xing, F., and Brunton, S. L., "Multiresolution Dynamic Mode Decomposition," SIAM Journal on Applied Dynamical Systems, Vol. 15, No. 2, 2016, pp. 713-735. https://doi.org/10.1137/15M1023543 
This article has been cited by:

1. B. Zebiri, A. Piquet, A. Hadjadj. 2021. On the use of a two-layer model for large-eddy simulations of supersonic boundary layers with separation. International Journal of Heat and Fluid Flow 90, 108821. [Crossref]

2. Kuangshi Chen, Jinglei Xu, Qihao Qin, Weijian Xiong. 2021. Design of Thrust-Optimized Scramjet Nozzle and Its Concise Estimation Method. AIAA Journal 59:6, 2014-2026. [Abstract] [Full Text] [PDF] [PDF Plus]

3. B. Zebiri, A. Piquet, A. Hadjadj. 2020. Analysis of shock-wave unsteadiness in conical supersonic nozzles. Aerospace Science and Technology 105, 106060. [Crossref]

4. M. Chaudhary, T. V. Krishna, Soumya R. Nanda, S. K. Karthick, A. Khan, A. De, Ibrahim M. Sugarno. 2020. On the fluidic behavior of an over-expanded planar plug nozzle under lateral confinement. Physics of Fluids 32:8, 086106. [Crossref] 